\title{
Maligne Hauterkrankungen bei organtransplantierten Patienten
}

Göksu, Y ; Hofbauer, G ; Serra, A ; Nowak, A

DOI: https://doi.org/10.1024/1661-8157/a000821

Other titles: Malignant skin diseases in organ transplant patients

Posted at the Zurich Open Repository and Archive, University of Zurich ZORA URL: https://doi.org/10.5167/uzh-63902

Journal Article

Originally published at:

Göksu, Y; Hofbauer, G; Serra, A; Nowak, A (2012). Maligne Hauterkrankungen bei organtransplantierten Patienten. Praxis, 101(2):75-83.

DOI: https://doi.org/10.1024/1661-8157/a000821 


\section{Maligne Hauterkrankungen bei organtransplantierten Patienten}

\section{Einleitung((Ü1))}

In den letzten Jahrzehnten kam es dank den chirurgischen und pharmakologischen Fortschritten zur Verbesserung des Patienten- und Transplantatüberlebens und damit zur Zunahme der Anzahl von organtransplantierten Patienten. Um eine Abstossungsreaktion nach Transplantation zu vermeiden, ist eine lebenslange immunsuppressive Therapie erforderlich. Mit zunehmender Prävalenz von LangzeitOrgantransplantierten Patienten gewinnen dermatologische Probleme wie Neoplasien und kutane Infektionen immer mehr an Bedeutung.Welt- und europaweit wurden kollaborative Organisationen gegründet, in denen Dermatologen, Nephrologen und Transplantationsspezialisten sich zusammen für die Früherkennung und rechtzeitige Behandlung von Hauterkrankungen bei Organtransplantierten Patienten stark machen. In der Schweiz operiert die «Swiss Society for Dermatology and Venerology (SGDV) working group for organ transplantation». Die Prinzipien der dermatologischen Betreuung von Schweizer organtransplantierten Patienten sind in der Arbeit von Hofbauer et a.I in der Zeitschrift Swiss Medical Weekly zusammengefasst [1]. Das Ziel dieser Arbeit ist es, eine kurze Übersicht über die häufigsten Hauttumorarten darzustellen, die dermatologische Betreuung vor und nach der Transplantation zu erläutern und die Bedeutung vorbeugender Massnahmen hervorzuheben.

\section{Im Artikel verwendete Abkürzungen}

CD4

Cluster of differentiation 4

$\mathrm{CNI}$ Calcineurin-Inhibitoren

mTOR Mammalian target of rapamycin

SCC Spinozelluläres Karzinom

TGF $\beta$ Transforming growth factor beta

VEGF Vascular endothelial growth factor

HPV Humanes Papilloma-Virus

HHV Humanes Herpesvirus 
Pathophysiolgie der Tumorentstehung

\section{Onkogene Faktoren ((Ü1))}

\section{Ultraviolettes Licht ((Ü2))}

Sonnenlicht trägt entscheidend zur Entwicklung des spinozellulären Karzinoms bei. UV-Licht schädigt direkt die Zell-DNA. Der Sonnenschutz ist daher die wichtigste Primärprävention. Systemische Chemoprevention mit Melanocortin-Rezeptor-Agonisten wird momentan in klinischen Studien erprobt [2].

\section{Immunsuppressiva((Ü2))}

Die Tumorinzidenz steigt mit der Intensität und Länge der Immunsuppression. Studien fanden eine negative Korrelation zwischen der Anzahl der CD4-Zellen und der Hauttumor-Inzidenz [2,5]. Patienten mit dreifacher Immunsuppressiva-Kombination entwickeln Hauttumoren dreimal häufiger als diejenigen mit ZweifachImmunsuppression. Repetitive Transplantatabstossungs-Reaktionen sind wegen der Notwendigkeit intensiver Immunsuppression mit höherer Hauttumorinzidenz assoziiert. Transplantierte mit moderaterer Immunsuppression dagegen, z.B. Lebertransplantierte oder Empfänger einer Lebendnierenspende, entwickeln wesentlich seltener maligne Hauttumoren [6].

Azathioprin erhöht die Sensitivität gegenüber UV-Licht und verursacht einen mutagenen oxidativen DNASchaden. In Kürze wird eine Studie erscheinen, die gezeigt hat, dass Umstellung von Azathioprin auf Mycophenolat-Mofetil die Photosensivität der Haut deutlich reduziert [7]. Calcineurin-Inhibitoren (CNI) erhöhen TGF $\beta$ - und VEGF-Spiegel und treiben auch direkt Keratinozyten zur Krebsentwicklung durch den kürzlich beschriebenen Transkriptionsfaktor [8]. In experimentellen Studien begünstigte Cyclosporin die Umwandlung von nicht-invasiven Epithelzellen in die invasive Form beim Adenokarzinom in vitro und führten zum Tumorwachstum verschiedener Zelllinien in vivo [9]. Tacrolimus scheint eine ähnliche onkogene Potenz zu besitzen.

\section{Humanes Papilloma-Virus ((Ü2))}

Dieses Virus spielt eine wahrscheinlich wichtige, aber noch nicht komplett verstandene Rolle in der Entstehung des spinozellulären Karzinoms. HPV wird häufig in viralen Warzen und den verschiedenen Stufen des spinozellulären Karzinoms gefunden, weshalb man HPV eine tumorfördernde Wirkung zuschreibt. Experimentelle Studien zeigen, dass virusspezifische Proteine die DNA-Reparatur nach UV-Licht induziertem Schaden beeinträchtigen und die Apoptose lichtgeschädigter Zellen herabsetzen [2,3]. Eine australische Forschungsgruppe entwickelt gegenwärtig eine Interventionsstudie mit der HPV-Impfung [4].

\section{Feldkanzerisierung ((Ü2))}

Ein Hauttumor kommt selten allein. Der Grund für hohe Rezidivrate ist u.a. die sogenannte Feldkanzerisierung. Darunter versteht man die Entwicklung von zahlreichen Nestern entarteter Keratinozyten als Resultat des flächenhaften Sonnenschadens. Man geht davon aus, dass diese neoplastischen Inseln sich in unterschiedlichen "Reifestadien" befinden und durch onkogene Stimulatoren beschleunigt werden können. 
Einteilung der Hauttumoren ((Ü2)

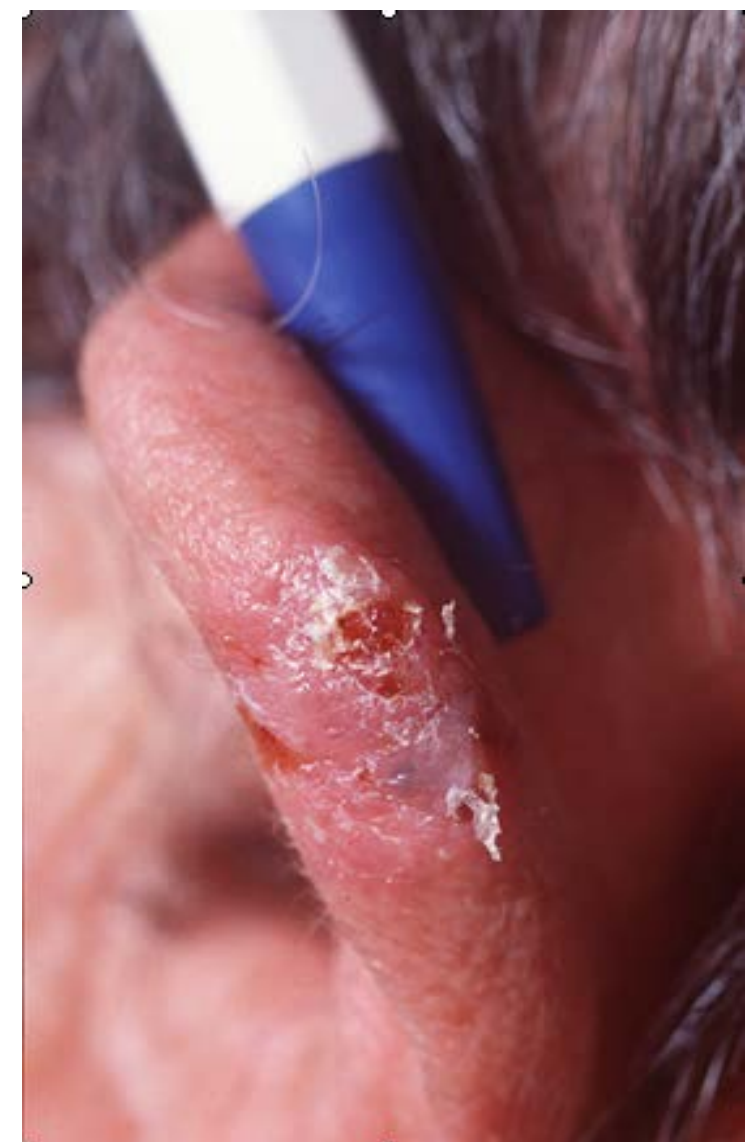

Abb. 1: Spinozelluläres Karzinom an der Ohrhelix links.

\section{Spinozelluläres Karzinom ((Ü2))}

Das spinozelluläre Karzinom (Abb. 1) ist der häufigste maligne Hauttumor bei organtransplantierten Patienten. Die Inzidenz bei Transplantierten ist 60-100mal höher als diejenige der altersentsprechenden Allgemeinbevölkerung. Das SCC entsteht aus atypischen Keratinozyten der sonnenbeschädigten Haut. Die intraepithelialen Vorläuferläsionen sind aktinische Keratosen und Morbus Bowen. 


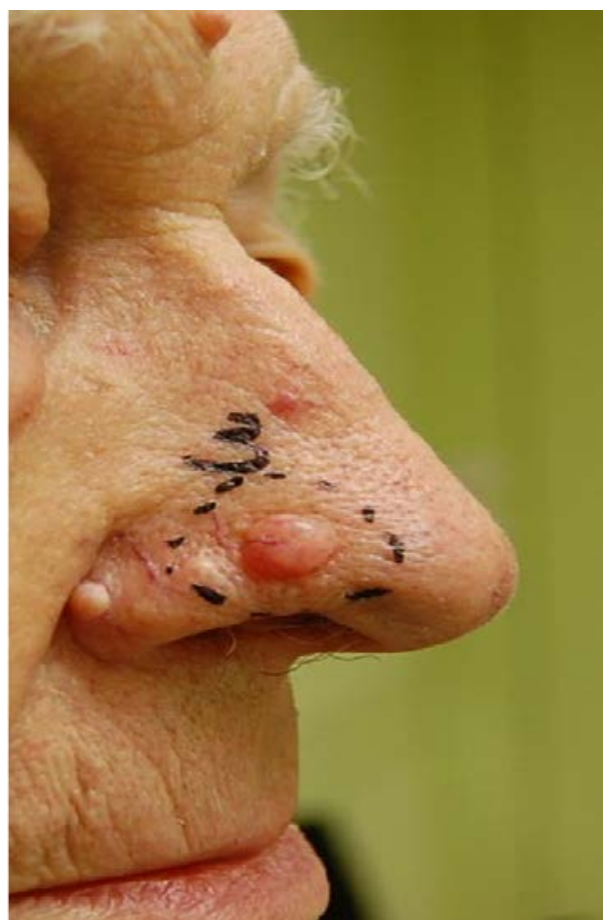

Abb. 2: Basalzellkarzinom Retroaurikullär

\section{Basalzellkarzinom ((Ü2))}

Das Basalzellkarzinom (Abb. 2) ist der zweithäufigste Tumor-Typ, welcher aus lokal infiltrierend-destruierenden Epithelzellen entsteht, nicht metastasiert und deshalb als «semimaligne» bezeichnet wird. Auch bei Basalzellkarzinom-Entstehung spielt chronischer Sonnenschaden durch UV-Licht eine wichtige karzinogene Rolle.

\section{Malignes Melanom ((Ü2))}

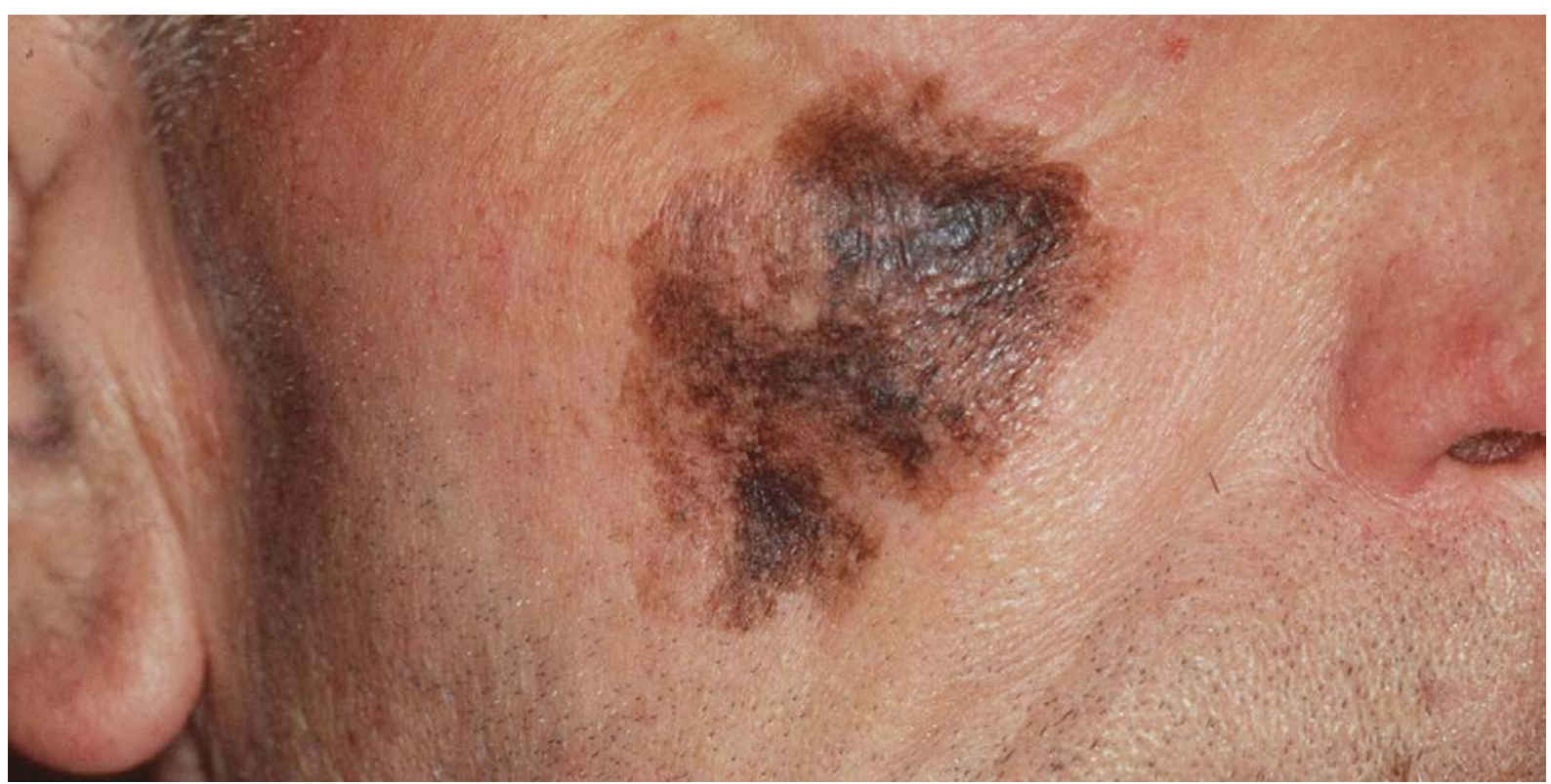

Abb. 3: Lentigo maligna Melanom an der Wange rechts, ein typischerweise auf chronisch sonnengeschädigter Haut entstehender Tumor. 
Das Melanom (Abb. 3) ist eine bösartige Neubildung aus Melanozyten der Haut. Durch frühzeitige Metastasierung ist dieser Tumor besonders bösartig. Es ist umstritten, ob die Melanom-Inzidenz bei organtransplantierten Patienten höher ist als in Allgemeinbevölkerung: sie wird von «nicht erhöht» «bis zu „8-fach erhöht» geschätzt.

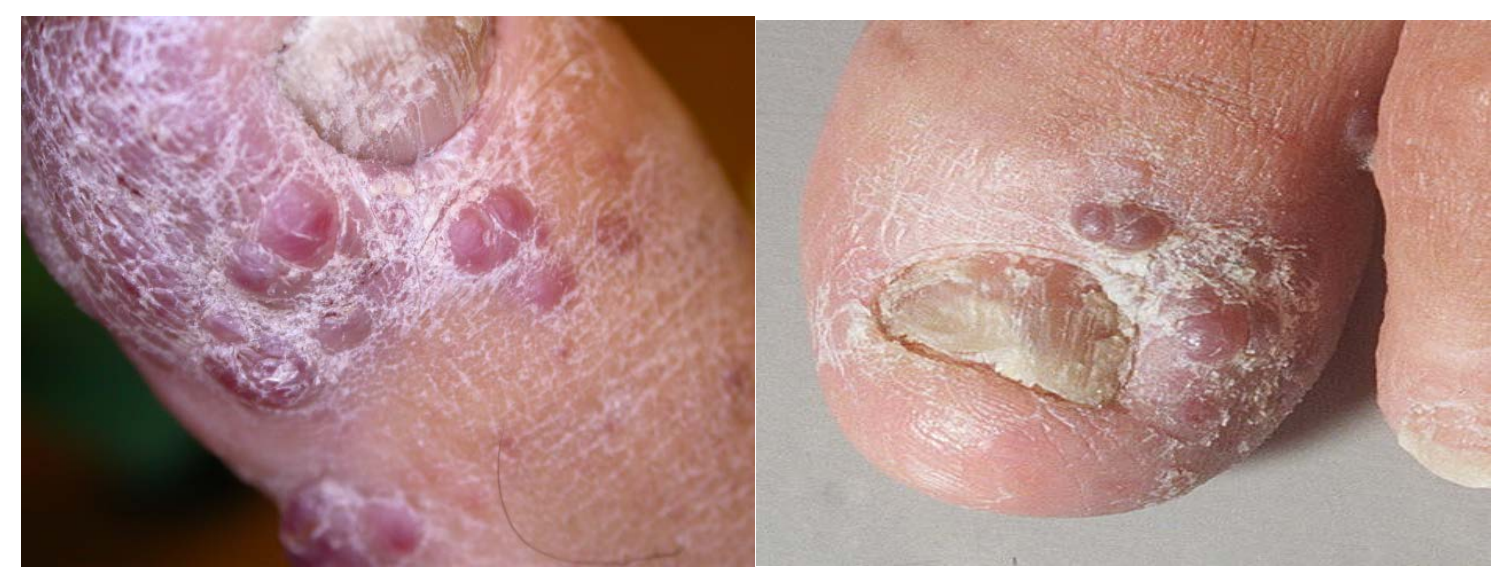

Abb. 4: Kaposi Sarkom unter Immunsuppression mit Ciclosporin A (links); 1 Jahr nach Umstellung auf Everolimus (rechts).

\section{Kaposi Sarkom ((Ü2))}

Das Kaposi Sarkom (Abb. 4) äussert sich durch das Auftreten von braun-bläulichen Tumorknoten an der Haut, Schleimhaut, aber auch im Darm, in der Lunge und anderen Organen. Dabei handelt es sich um eine von Blutgefässen ausgehende Neubildung mit multizentrischem Beginn und langsamer Ausbreitung. Der häufige Nachweis von Herpes-Virus Typ 8 spricht für eine virusinduzierte Neoplasie. Die Wahrscheinlichkeit, an einem Kaposi Sarkom zu erkranken, ist bei Organtransplantierten 400 bis 500-fach höher im Vergleich zur Allgemeinbevölkerung. Die Prävalenz des Kaposi Sarkoms nach Organtransplantation ist abhängig von der Prävalenz der HHV8-Infektion in der Bevölkerung und ist je nach ethnischem Hintergrund unterschiedlich, z.B. USA und Europa ca. 0,4\%, Saudi Arabien ca. 5\%. In Europa sind vor allem Menschen süd- und osteuropäischer Herkunft von diesem Tumor betroffen.

\section{Seltene Tumore ((Ü2))}

Das Merkel-Zell-Karzinom ist ein hochmaligner kutaner Tumor, der von neuroendokrinen Merkel-Zellen ausgeht, in dessen Entstehung das kürzlich entdeckte Merkelzellpolyomavirus ursächlich beteiligt sein dürfte. Zu den anderen seltenen Hauttumoren zählen atypische Fibroxanthome, Angiosarkome, Leiomyosarkome und kutane Tund B-Zell-Lymphome. 
Aktuelle Hauterkrankungen sollten erkannt und behandelt, das Risiko für künftige Hautmalignome unter Immunsuppression evaluiert und die Patienten instruiert werden.

\section{Screening ((Ü2))}

Eine dermatologische Untersuchung (inklusive oral und anogenital) ist bereits vor der Transplantation indiziert. Anamnese über die kumulative UV-Licht-Exposition, Häufigkeit der Sonnenbrände, Familien- und persönliche Anamnese über mögliche vorausgegangene maligne und benigne Hauttumoren sollte erhoben werden. Von Wichtigkeit ist ebenfalls die frühere Exposition gegenüber Immunsuppressiva, z.B. wegen einer bereits stattgehabten Transplantation oder Therapie von Autoimmunerkrankungen wie Vaskulitis oder Glomerulonephritis. Angeborene und erworbene Immundefekte sollen berücksichtigt werden. Hautwarzen und intraepitheliale Läsionen wie Morbus Bowen und Aktinische Keratosen sollten vor der Transplantation bereits behandelt und invasive Tumoren exzidiert werden.

\section{Patientenschulung ((Ü2))}

Der Patient sollte informiert werden, dass sein Hautkrebsrisiko nach der Transplantation erhöht sein wird. Der konsequente Sonnenschutz ist besonders wichtig. Patienten sollten Sonnenexposition, insbesondere zwischen 11 und 15 Uhr, vermeiden, einen Hut mit breitem Rand, eine Sonnenbrille, langärmlige Oberteile und Hosen tragen. Zudem sollten Patienten eine Sonnencreme mit UV-Schutzfaktor von mindestens 50 auf die sonnenexponierten Hautpartien wie Gesicht, Ohren, Nacken und Handrücken auftragen. Dies sollte täglich und nicht nur bei sonnigem Wetter erfolgen. Solarien sollten vermieden werden.

Patienten sollten angehalten werden, monatliche Inspektionen der eigenen Haut vorzunehmen. Es sollte nach neuen Hautveränderungen wie Rötungen, Schuppungen, Blutungen oder wachsenden Läsionen Ausschau gehalten werden. Patienten mit dermatologischem Tumorleiden in der Vorgeschichte sollten in monatlichen Abständen ihre Lymphknoten tasten. Das Wachstum einer Läsion oder nicht heilende Wunden über vier Wochen hinaus sollten dringend dermatologisch beurteilt werden.

Die Patientenschulung sollte regelmässig wiederholt werden. Die mündliche Informationsvermittlung sollte schriftlich mittels Broschüren oder Internet unterstützt werden. Diese Informationen finden sich auch online unter www.dermaguard.ch.

Management und Therapie der malignen Hauttumore nach Transplantation ((Ü1))

\section{Dermatologische Konsilien ((ü2))}

Organtransplantierte Patienten sollten jährlich dermatologisch beurteilt werden. Bei Risikopatienten müssen die Untersuchungen in kürzeren Intervallen erfolgen. In der dermatologischen Konsultation sollte eine genaue Anamnese hinsichtlich Hautneubildungen und Einhalten von Sonnenschutzmassnahmen erhoben werden. Eine komplette Haut- und Schleimhautuntersuchung sowie Lymphknotenpalpation gehört ebenfalls dazu. Eine diagnostische Biopsie von verdächtigen Hautläsionen sollte niederschwellig durchgeführt werden.

\section{Spezifische Tumortherapie ((Ü2))}


Die Sekundärprävention ist für in-situ spinozelluläres Karzinom besonders wichtig. Die bereits entstandenen Läsionen sollten nach Möglichkeit früh erkannt und früh therapiert werden. Das Phänomen der Feldkanzerisierung mit grösseren Arealen sonnengeschädigter Haut ist typisch für diese Tumorart. Zu den Behandlungsmöglichkeiten der Feldkanzerisierung gehören topisches 5-Fluorouracil, Imiquimod und die photodynamische Therapie. Wegen additiver DNS-Schädigung ist Strahlentherapie nicht empfohlen. Ein invasives spinozelluläres Karzinom sollte komplett und möglichst frühzeitig mit einem Sicherheitsabstand von 4-6 mm exzidiert werden, um Metastasenbildung vorzubeugen. Im Falle von fortgeschrittenem invasivem Wachstum mit Lymphknoten- und Organbefall sollte eine individuelle Therapie interdisziplinär herausgearbeitet werden. Die Therapie des Basalzellkarzinoms bei immunsupprimierten Patienten erfolgt gemäss den Richtlinien der Allgemeinbevölkerung und besteht ebenfalls vor allem in der Exzision. Auch hier ist die Strahlentherapie wegen der additiven DNS-Schädigung nicht empfohlen. Die Behandlung des Melanoms unterliegt den Richtlinien der Allgemeinbevölkerung und beinhaltet die Exzision in möglichst frühen Stadien. Für die Diagnose eines Kaposi Sarkoms ist eine bioptische Bestätigung wegen möglicher variabler klinischer Erscheinungsbilder wichtig. Ein multifokaler Befall von Haut und Schleimhaut ist nicht selten und ist oft mit viszeralem Befall assoziiert. Die Therapie des Kaposi Sarkoms ist primär nicht operativ. Die Reduktion der Immunsuppression und Umstellung von Calcineurininhibitoren auf mTOR-Inhibitoren (Mammalian target of rapamycin inhibitors) z.B. Rapamune ${ }^{\circledR}$ führen gemäss aktueller Studienlage zu Tumorstabilisierung und sogar Tumorreduktion bei nierentransplantierten Patienten [10].

\section{Immunsuppression((Ü2))}

Alle Immunsupressiva tragen zu Entstehung der Hautmalignome bei. Die effektivste Methode, die Tumorinzidenz zu senken, ist die Reduktion der Immunsuppression. Die Wirkstoffgruppe der mTOR-Inhibitoren zeigte antiproliferative Effekte in vitro und in vivo. Derzeit laufen multizentrische prospektive Studien, die durch Umstellung von CNI auf mTOR-Inhibitoren präliminär eine Senkung der Inzidenz von SCC um ca. 50\% andeuten. Eine endgültige Publikation gilt es jedoch noch abzuwarten. Ein Wechsel der immunsuppressiven Therapie muss zwingend individuell, am besten multidisziplinär diskutiert werden.

\section{Systemische Chemoprävention ((Ü2))}

Systemische Retinoide wie Acitretin (Neotigason®) zeigten bis zu 85\% Reduktion der SCC-Incidenz. Indiziert ist das Medikament bei Patienten mit stabiler Transplantatfunktion bei moderatem bis schwerem SCC-Befall der Haut (ein bis drei SCC jährlich). Die Anfangsdosis beträgt meistens $10 \mathrm{mg}$ am Morgen jeden zweiten Tag für drei Wochen. Bei Verträglichkeit wird auf $10 \mathrm{mg}$ täglich für weitere drei Wochen und dann während der darauf folgenden 3 Wochen alternierend zu $10 \mathrm{mg}$ und $25 \mathrm{mg}$ täglich aufdosiert. Die Erhaltungsdosis beträgt meistens $25 \mathrm{mg}$ jeden Morgen. Die häufigsten Nebenwirkungen sind Xerosis cutis, Mundtrockenheit, ein bei Dosisreduktion reversibler Haarausfall, Anstieg von Transaminasen, Cholesterin und Triglyzeriden sowie Teratogenität.

\section{Schlussfolgerung((Ü2))}

Wegen der steigenden Anzahl organtransplantierter Patienten und der dadurch notwendigen Immunsuppression mehren sich die dermatologischen Probleme wie maligne Hautneoplasien. Eine interdisziplinäre Betreuung dieser Patienten ist wichtig und ein potentieller Beitrag des betreuenden Grundversorgers ist wertvoll, insbesondere in der Früherkennung der malignen Hautveränderungen und der Patientenschulung. 


\section{Fallbericht zu den malignen Hauterkrankungen bei organtransplantierten}

\section{Patienten}

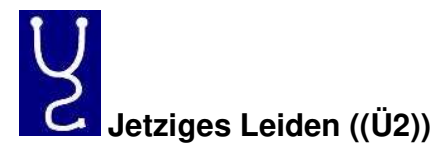

Ein 61-jähriger nierentransplantierter Patient stellte sich in der dermatologischen Sprechstunde vor wegen multiplen teilweise neu aufgetretenen teilweise schon seit Jahren bestehenden rötlich tastbaren, zum Teil schuppenden Maculae und Papeln sowie kleinen schmerzlosen Erosionen der sonnenexponierten Kopfhaut (Abb. 4) sowie seitlich an Ohrmuschel, im Dekolleté, an den Unterarmen und diffus verstreut am ganzen Oberkörper. An der linken Flanke bestand neu seit drei Monaten eine an Grösse zunehmende dunkelbraune, stellenweise schwarze, unregelmässig begrenzte teils erhabene Macula.

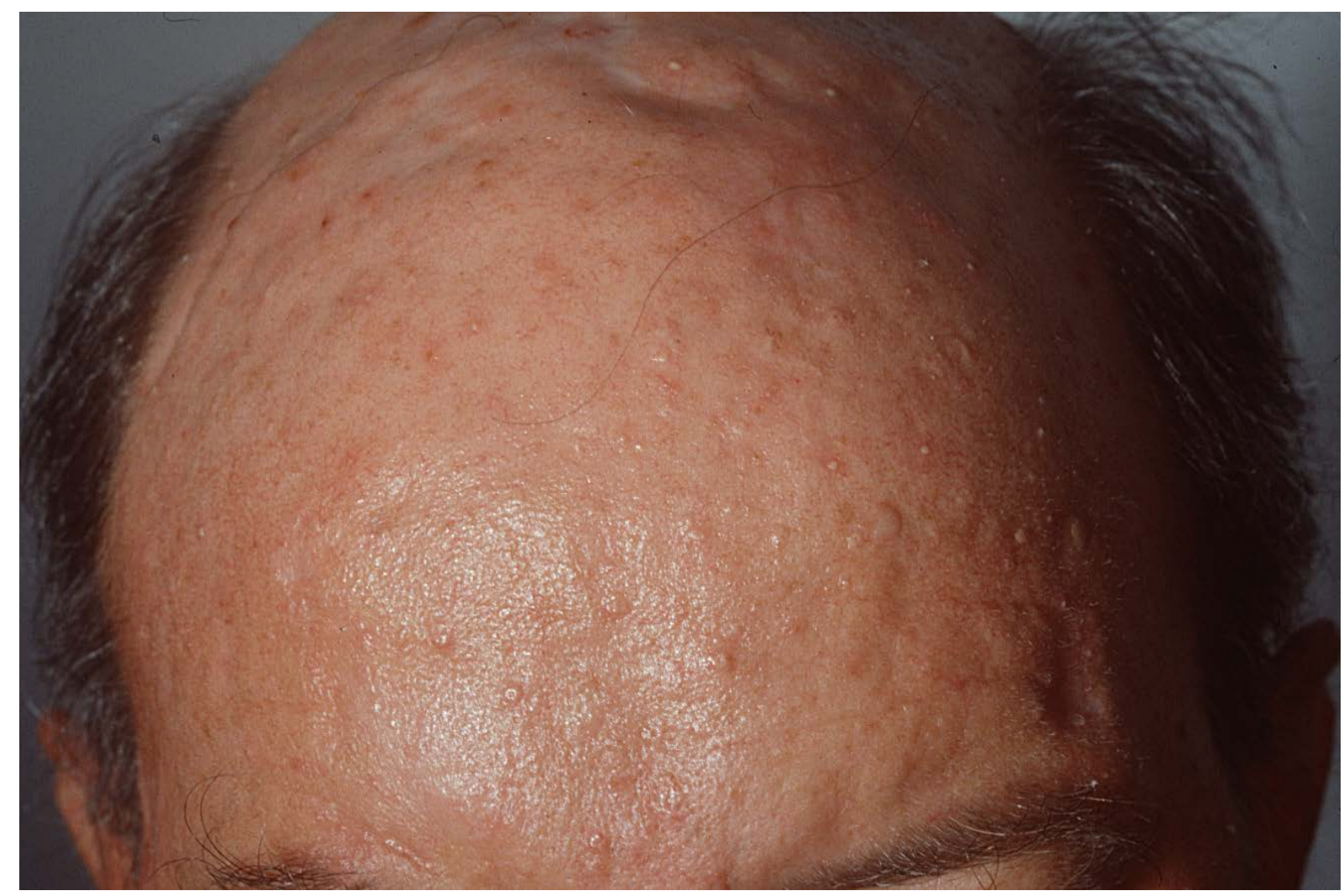

Abb. 4: Fallbericht Kopfbefund.

\section{Persönliche Anamnese ((Ü2))}

Mit 31 Jahren erkrankte der Patient an einer fokal sklerosierenden Glomerulonephritis mit nephrotischem Syndrom und musste zunächst fünf Jahre drei Mal wöchentlich jeweils vier Stunden zur Hämodialyse. Mit 36 Jahren wurde er erfolgreich nierentransplantiert und steht seither unter dreifacher Immunsuppression mit Ciclosporin A, Azathioprin und Prednison. Ca. sechs Monate nach der Transplantation verspürte der Patient endlich wieder das Freiheitsgefühl, nicht mehr an die Dialyse gebunden zu sein. Er erfülte sich seinen Lebenstraum, indem er nach Spanien auswanderte und ein Segelboot kaufte. Angeln und Segeln wurden zu seinen Hobbys. Zwei Monate im Jahr verbrachte er zudem auf Kreuzfahrten im karibischen Meer oder in der Südsee, wo er nicht selten die frische Luft am Sonnendeck genoss. Gelegentlich stellte er sich bei seinem spanischen Hausarzt vor, um Medikamentenspiegelmessungen und die Dosisanpassung der Immunsuppression 
zu veranlassen. Andere inm empfohlene Nachsorgeuntersuchungen wie dermatologische Kontrollen konnte er jedoch aus zeitlichen Gründen nicht wahrnehmen.

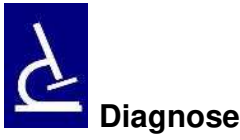

Eine sorgfältige dermatologische Untersuchung enthülte zusätzlich multiple weitere Hautläsionen. Klinisch und bioptisch diagnostizierte man ein Melanom vom nicht sicher klassifizierbarem Typ, Breslow-Index 0,57 mm an der linken Flanke, spinozelluläre Karzinome sowie Basalzellkarzinome und aktinische Keratosen am Capillitium, ein spinozelluläres Karzinom am Handrücken radial und ulnar links sowie am Hals lateral rechts und rechts parasternal, ein Basalzellkarzinom parasternal links, sternal links, Oberarm links und Unterarm links. Zusätzlich fanden sich aktinische Keratosen am Antitragus, hochfrontal und frontal rechts. Multiple Verrucae vulgares. Zwei atypische Nevuszellnävi paravertebral BWS rechts. Ein atypischer melanozytärer Nävus am Abdomen links.

Abb. 5: Fallbericht Oberkörperbefund ((Autor: Bitte Abbildung an uns schicken))

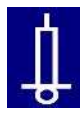

Es folgte eine Exzision von elf Basalzellkarzinomen und neun spinozellulären Karzinomen sowie des Melanoms; multiple Kryotherapien bei Verrucae vulgares, Nävi und aktinischen Keratosen. Der Patient wurde über die Sonnenschutzmassnahmen informiert und in der dermatologischen Selbstinspektion geschult. Jedoch wurden viele weitere dermatologische Vorstellungen wegen neu aufgetretenen Hauttumoren, insbesondere im Bereich der ehemals sonnenexponierten Hautareale, während der folgenden Jahren nötig. Deshalb erfolgten eine Reduktion der Sandimmundosis sowie eine Umstellung von Azathioprin auf Mycophenolat Mofetil, gefolgt vom langsamen Ausschleichen des Prednisons.

Wegen der zunehmenden chronischen Transplantatglomerulopathie und beginnender Urämie wurde der Patient dialysepflichtig. Wegen der stark eingeschränkten Transplantatfunktion und karzinogenen Wirkung der Immunsuppressiva wurde schliesslich das Nierentransplantat entfernt. Der Patient kam erneut zur chronischintermittierenden Hämodialyse dreimal pro Woche.

Eine Neuaufnahme auf die Transplantationsliste wird bis zur Stabilisierung der dermatologischen Befunde nicht empfohlen. 


\section{Fragen zu den malignen Hauterkrankungen bei organtransplantierten Patienten}

Frage 1

Welche maligne Hautveränderung kommt am häufigsten bei Organtransplantierten vor? (Einfachauswahl)
a) Melanom
b) Spinozelluläres Karzinom
c) Basalzellkarzinom
d) Kaposi Sarkom

Frage 2

Was beinhaltet die dermatologische Prätransplantationsabklärung? (Mehrfachauswahl)
a) Vollständige Anamnese
b) Phototherapie vor Transplantation
c) Dermatologische Untersuchung
d) Schulung bezüglich Sonnenschutz
e) Exzision aller Nävuszellnävi

Frage 3

Welche Wirkstoffe werden als systemische Chemoprophylaxe gegen das spinozelluläre Karzinom bei Patienten nach Organtransplantation eingesetzt? (Einfachauswahl)
a) Azathioprin
b) Kalzineurininhibitoren
c) mTOR-Inhibitoren
d) Mycophenolat
e) Retinoide

Frage 4

Welches sind die kutanen Warnzeichen beim immunsupprimierten Patienten? (Mehrfachauswahl)
a) Persistierende Rötung
b) Grössenzunahme einer Hautläsion
c) Sandpapierartige Schuppung
d) Haarausfall
e) Nicht heilende Wunden

Frage 5

Was gehört zur Prävention von malignen Hauterkrankungen durch die transplantierten Patienten?

(Einfachauswahl)

a)Tragen von Bermudas und Muskelshirts

b) Sonnencreme 50+ nur bei sonnigem Wetter

c) Schonende Bräunung der Haut im Solarium in einschleichender Dosierung

d) Selbstinspektion der Haut in halbjährlichen Abständen 
e) Jährliche dermatologische Kontrollen 
((CME))

\section{Antworten zu den Fragen aus PRAXIS Nr.2 «Maligne Hauterkrankungen bei organtransplantierten Patienten»}

\section{Frage 1}

\section{Richtig ist Antwort b)}

Das spinozelluläre Karzinom ist der häufigste maligne Hauttumor bei Organtransplantierten. Die Inzidenz bei Transplantierten ist 60-100mal höher als bei der altersentsprechenden Allgemeinbevölkerung.

\section{Frage 2}

Richtig sind die Antworten a), c), d)

Ad a) Bei der dermatologischen Prätransplantationsabklärung wird das posttransplantäre Hauttumorrisiko abgeschätzt. Bei positiver Eigen- oder Familienanamnese steigt das Hauttumorrisiko.

Ad b) UV-Licht ist ein bedeutender Risikofaktor für die Entstehung von Hauttumoren und sollte daher vermieden werden.

Ad c) In einer sorgfältigen dermatologischen Untersuchung inklusive Genitalregion und Schleimhäute werden Hauttumore gesucht.

Ad d) Die Primärprävention ist besonders wichtig. Daher müssen die Patienten durch Dermatologen und Grundversorger beim Sonnenschutz geschult werden.

Ad e) Bei weitem nicht alle Naevi sind potenzielle Hauttumorvorstufen.

\section{Frage 3}

\section{Richtig ist Antwort e)}

Systemische Retinoide wie Acitretin (Neotigason®) zeigten eine Reduktion der SCC-Inzidenz von bis zu $85 \%$.

\section{Frage 4}

Richtig sind Antworten a), b), c), e)

Ad a) Neu entstandene Rötungen können ein Hinweis auf Hautkrebsvorstufen sein (z.B. Aktinische Keratose).

Ad b) und e) Wachstum von Hautläsionen oder nicht heilende Wunden sind grundsätzlich tumorverdächtig.

Ad c) Sandpapierartige oder reibeisenartige Schuppung auf geröteter Haut ist typisch für aktinische Keratosen.

Ad d) Haarausfall ist nicht malignitätsverdächtig und kann z.B eine medikamentöse Nebenwirkung von.

Cyclosporin- oder Retinoideinnahme sein.

\section{Frage 5}

\section{Richtig ist Antwort e)}

Dermatologische Kontrollen sollten jährlich erfolgen, bei Risikopatienten häufiger.

Ad a) Patienten sollten einen Hut mit breitem Rand, eine Sonnenbrille, langärmlige Oberteile und Hosen tragen.

Ad b) Sonnencreme sollte IMMER, auch bei Regen aufgetragen werden.

Ad c) Auch kleinste Mengen an UV-Strahlen sind potentiell schädlich.

Ad d) Die Selbstinspektion der Haut sollte mindestens monatlich erfolgen. 


\section{Autoren}

Klinik und Poliklinik für Innere Medizin ${ }^{1}$, Klinik für Dermatologie ${ }^{2}$, Klinik für Nephrologie ${ }^{3}$, Universitätsspital Zürich

${ }^{1}$ Yasemin Göksu, ${ }^{2}$ Günther Hofbauer, ${ }^{3}$ Andreas Serra, ${ }^{1}$ Albina Nowak

\section{((Autor: bitte Korrespondenzadresse angeben))}

\section{Bibliographie}

1. Hofbauer GF, Anliker M, Arnold A, et al.: Swiss clinical practice guidelines for skin cancer in organ transplant recipients. Swiss Med Wkly 2009;29-30:407-415.

2. Hofbauer GF, Bouwes Bavinck JN, Euvrard S: Organ transplantation and skin cancer: basic problems and new perspectives. Exp Dermatol 2010;6:473-482.

3. Bavinck JNB, Feltkamp MCW: Milk of human kindness? HAMLET, human papillomavirus, and warts. New Engl J Med 2004;26:2639-2642.

4. Grose S. HPV researchers set sights on preventing skin cancers. Nat Med 2009;1:5.

5. Ducloux D, Carron PL, Rebibou JM: CD4 lymphocytopenia as a risk factor for skin cancers in renal transplant recipients. Transplantation 1998;9:1270-1272.

6. Euvrard S, Kanitakis J, Claudy A: Skin cancers after organ transplantation - Reply. New Engl J Med 2003;6:613-614.

7. Günther FL, Hofbauer NRA, Catherine A, Harwood, Jane M. McGregor et al.: Reversal of UVA skin photosensitivity and DNA damage in kidney transplant recipients by replacing azathioprine. American Journal of Transplantation (in press).

8. Wu X, Nguyen BC, Dzjunycz P, Chang S, Brooks Y, Lefort K, Hofbauer GF, Dotto GP: Opposing roles for calcineurin and ATF3 in squamous skin cancer.

Nature 2010; 465:368-372.

9. Hojo M, Morimoto T, Maluccio M: Cyclosporine induces cancer progression by a cell-autonomous mechanism. Nature 1999;6719:530-534.

10. Stallone G, Schena A, Infante B: Sirolimus for Kaposi's sarcoma in renal-transplant recipients. N Engl J Med 2005;13:1317-1323. 